\section{High levels of long chain fatty acids associated with decreased risk of prostate cancer}

Animal studies that have investigated the relationship between fatty acid intake and the risk of prostate cancer have led to the assumption that $\mathrm{n}-3$ fatty acids inhibit prostate cancer growth while $\mathrm{n}-6$ fatty acids have the opposite effect; however, there is no epidemiological evidence to support this assumption. Chavarro and colleagues evaluated the association between blood levels of $n-3$ and $n-6$ polyunsaturated fatty acids and risk of prostate cancer.

The authors based their analysis on blood samples taken from 476 prostate cancer cases that occurred during the 13-year follow-up of the Physician's Health Study. Whole-blood fatty acid levels were recorded for these men. Regression models were used to estimate the relative risk of prostate cancer at different levels of specific fatty acids expressed as a percentage of the total blood fatty acid concentration.

The results demonstrated an inverse relationship between blood levels of all long chain $n-3$ fatty acids and linoleic acid with overall prostate cancer risk. Direct associations between prostate cancer and blood levels of $\gamma$-linolenic and dihomo- $\gamma$-linolenic acids (both metabolites of linoleic acid) were noted. Blood levels of $a$-linolenic acid and arachidonic acid were not related to prostate cancer.

These findings suggest that the risk of prostate cancer is unlikely to be increased by the intake of polyunsaturated fatty acids. The intake of long chain n-3 fatty and linoleic acids could actually decrease the risk of prostate cancer. Further studies are needed to confirm this.

Original article Chavarro J et al. (2007) A prospective study of polyunsaturated fatty acid levels in blood and prostate cancer risk. Cancer Epidemiol Biomarkers Prev 16: 1364-1370

\section{Autologous myoblasts and fibroblasts show promise for treatment of stress incontinence}

An Austrian study has shown that injection of autologous myoblasts and fibroblasts is much more effective than collagen injection for the treatment of urinary stress incontinence.
The randomized, controlled trial involved 63 females (age 36-84 years) with urinary stress incontinence. A ratio of 2:1 was employed, such that 42 women were allocated to receive injections of autologous cells whereas 21 women received collagen. Patients in the autologouscell group provided a muscle biopsy sample from which myoblasts and fibroblasts were isolated and cultured; a transurethral ultrasonography-guided device was used to inject a myoblast suspension into the rhabdosphincter and a fibroblast-collagen suspension into the urethral submucosa. The other group underwent endoscopic transurethral injection of collagen.

At baseline, all women were completely incontinent. A year after treatment, 38 of 42 members of the group that had undergone autologous-cell injection were completely continent, in contrast to the collagen-treated group, of which only 2 of 21 were continent. Injection of the autologous cells increased the thickness and improved the contractility of the rhabdosphincter and led to regeneration of the urethral submucosa. None of the 63 patients reported adverse effects, and the positive outcome had not changed after a median follow-up period of 3 years.

Large, multicenter trials are required to assess whether injection of autologous cells should become the standard treatment for stress incontinence.

Original article Strasser H et al. (2007) Autologous myoblasts and fibroblasts versus collagen for treatment of stress urinary incontinence in women: a randomised controlled trial. Lancet 369: 2179-2186

\section{Dose-dependent association between bisphosphonates and jaw complications}

Bisphosphonates successfully treat bone metastases associated with certain cancers and bone loss caused by androgen deprivation therapy for prostate cancer, but bisphosphonate use has been associated with osteonecrosis of the jaw. Wilkinson et al. surveyed data from the Medicare-linked Surveillance, Epidemiology and End Results (SEER) database to investigate this association. The authors identified 14,349 cancer patients who were treated with intravenous pamidronate or zoledronic acid; these patients 